\title{
Parameter Settings for Reconstructing Binary Matrices from Fan-beam Projections
}

\author{
Antal Nagy and Attila Kuba \\ Department of Image Processing and Computer Graphics, University of Szeged, Hungary
}

\begin{abstract}
The problem of reconstruction of binary matrices from their fan-beam projections is studied. A fan-beam projection model is implemented and used in systematic experiments in order to determine the optimal parameter values for data acquisition and reconstruction algorithm. The fan-beam model, the reconstruction algorithm, the simulation experiments, and the results are discussed in the paper.
\end{abstract}

Keywords: discrete tomography, fan-beam projections, simulated annealing.

\section{Introduction}

Discrete tomography (DT) is used for reconstructing special objects consisting of a few types of known homogeneous materials. For example, if we know that the object is made of wood, then the space contains only two materials: wood and air. Such objects can be represented with two-valued functions. The knowledge of the discrete range of the function to be reconstructed can also reduce the number of necessary projections drastically. For a summary of theory and applications of DT see [1].

This paper deals with the reconstruction of binary matrices, i.e., the matrix elements can be either 0 or 1 . The reconstruction methods of binary matrices from parallel projections are available, i.e., when the sums of matrix elements along the lines parallel to given directions are given (e.g., row and column sums) is a well understood area of DT $[2,3]$. Surprisingly, there are few results published in connection with fanbeam projections $[4,5]$. A fan-beam projection

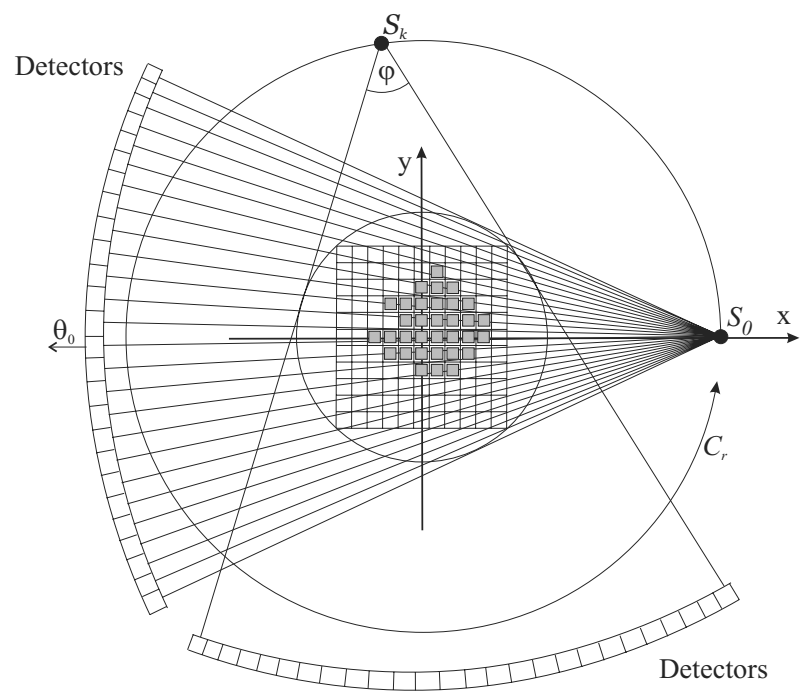

(a) Using line integrals

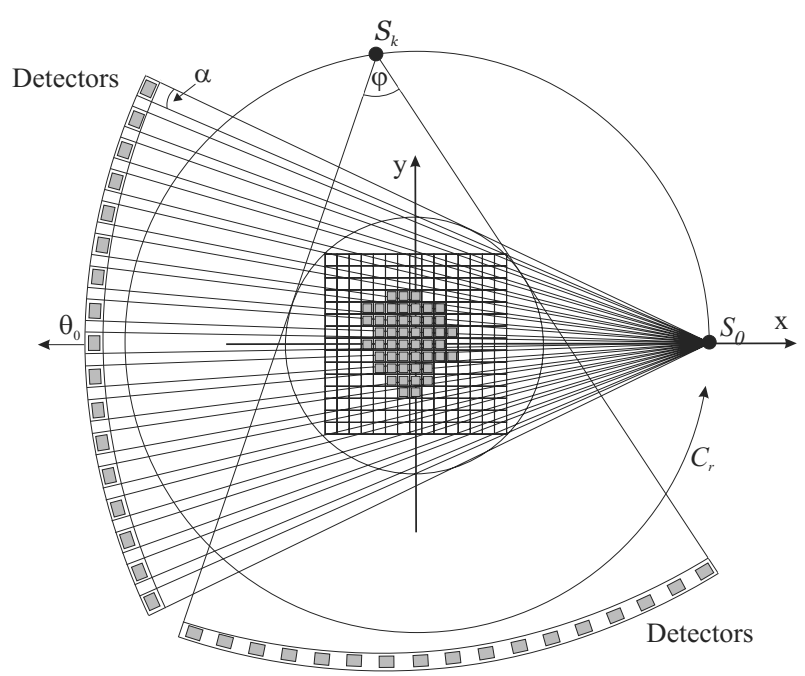

(b) Using strip integrals

Fig. 1. The geometry of our fan-beam model. 
is a collection of sums of matrix elements arranged in a fan-shaped area determined by two rays having the same source point (Fig. 1).

Most of the currently used CT scanners apply fan-beam projections. There are also other applications of tomography (e.g., non-destructive testing with X-rays [6] or neutrons [7]) where fan-beam projections are collected. At the same time, most of the papers about DT deal with parallel projections. We believe that fan-beam projections should play similarly important role in the applications of DT as in the case of classical (non-discrete) tomography. For this reason it is important to study the problems connected with fan-beam projections in DT (the only paper we found is [8]). Such studies can be interesting not only from the viewpoint of the implemented reconstruction algorithm (more generally, the software), but also from the viewpoint of the construction of the data acquisition system, i.e., the hardware of the DT.

This paper deals with the optimal setting of the geometric parameters in case of fan-beam projections. The method for this study is simulation. A software system is implemented which is able to simulate the collection of projections of binary matrices (2D objects), then to perform the reconstruction, and finally, to compare the reconstructed binary matrix with the original one. Simulation is suitable for studying the effects of different parameters of a complex system separately and for giving an estimate of the performance of a similar real system. In a previous work [9] we used this simulation system for studying the reconstruction algorithm. Now we investigate also the differences between the half-line integrals (Fig. 1(a)) and strip integrals (Fig. 1(b)) when changing the fan-beam geometric parameters (e.g. changing the number of sources and detector elements). A series of reconstructions is performed varying only one parameter while keeping all others fixed. The projections are computed analytically, according to the actual parameter setting. The measurement errors are simulated by additive random noise. As reconstruction algorithm a version of the random search optimization method of Simulated Annealing (SA) was implemented [10]. The reconstructed images were compared with the original image on the basis of the relative mean square error $[11,12]$.
The structure of the papers is as follows. In Section 2 the reconstruction problem is introduced with the necessary definitions and notation in the case of fan-beam projections. In Section 3 the DT reconstruction problem is reformulated as an optimization problem, which is solved by the method of SA. The results of our experiments and our discussions are detailed in Section 4. Finally, the last chapter gives the conclusions we obtained from our works.

\section{The Reconstruction Problem and Fan-beam Projections}

Let $f$ be an integrable real function on the $\mathbb{R}^{2}$ plane. Let $S$ be a point, called source point, and $v_{\theta}$ be a unit vector in the direction $\theta \in[0,2 \pi)$ on the plane. Consider the integrals of $f$ along the half-lines starting from $S$ in direction $v_{\theta}$ :

$$
[\mathcal{R} f](S, \theta)=\int_{0}^{\infty} f\left(S+u \cdot v_{\theta}\right) d u .
$$

The transformation defined by (1) is called the fan-beam projection of $f$ taken from the point $S$ in the direction $\theta$. Given a set $\mathcal{S}$ of source points, the reconstruction problem using fanbeam projections can be posed as follows.

\section{RECONSTRUCTION FB(S)}

Given: An integrable function, $g: \mathcal{S} \times[0,2 \pi) \rightarrow \mathbb{R}$.

Task: $\quad$ Construct a function $f$ such that

$$
[\mathcal{R} f](S, \theta)=g(S, \theta)
$$

for all $S \in \mathcal{S}$ for almost every $\theta \in[0,2 \pi)$.

In this paper we are interested in the reconstruction of special functions from fan-beam projections. Henceforth, let us suppose that the support of $f$ can be covered by a $n \times n$ regular lattice $W$ such that $f$ is constant on each $1 \times 1$ square of the lattice, namely $f$ can take the value of either 0 or 1 . That is, $f$ can be represented by a $n \times n$ binary matrix or, equivalently, by a vector $x \in\{0,1\}^{J}$ where $x_{j}$ denotes the $j$ th element of the matrix, say, in a row by row order $j=1,2, \ldots, J$ and $J=n^{2}$.

In the applications projections are taken only from a finite number of source points and the 
integrals are taken not along half-lines but on areas (let us call them fans) determined by two half-lines having angle $\alpha$ between them.

Let $S_{k}, k=1,2, \ldots, K$, denote the number of source points. They are situated along a circle $C_{r}=\left\{(x, y) \mid x^{2}+y^{2}=r^{2}\right\}$ around the origin $O$, where $r>0$ is large enough so that $W$ is in $C_{r}$. Furthermore, it is also usual that source points are distributed along $C_{r}$ uniformly, that is, $S_{k}=\left(r \cdot \cos \theta_{k}, r \cdot \sin \theta_{k}\right)$, where $\theta_{k}=\theta_{0}+(k-1) \cdot 2 \pi / K$ for all $k=1,2, \ldots, K$. The start angle $\theta_{0} \in[0,2 \pi)$ determines not only the position of the first, but of all source points. For example, the start angle $\theta_{0}=0$ means that the first source is in the intersection of circle $C_{r}$ and the positive part of axis $x$. The reason for the inclusion of the initial angle $\theta_{0}$ into the model is that since we usually have only a few (e.g., 2-4) source points, not only their number, but also their positions can have strong influence on the reconstruction, as we show later.

It is supposed that the integrals of the binary matrix are measured on a finite, say, $L$ number of $\alpha$ angle fans from each source point (Fig. 1). Fans are created in the same way from each source point. Fans from point $S_{k}$ are distributed uniformly between the half-lines starting form $S_{k}$ and touching the circle containing $W$. The angle between these two tangents is denoted by $\varphi$ (see Fig. 1). Therefore, all fans are determined uniquely by the parameters $r, L$, and $\alpha$.

The $i$ th fan-beam projection sample $b_{i}$, from $S_{k}$ can be described by the linear equation

$$
\sum_{j=1}^{J} a_{i j} x_{j}=b_{i}, \quad i=1,2, \ldots, I,
$$

where $a_{i j}$ denotes the common area between the $i$ th fan and the $j$ th unit square of $W$ and $I=K \cdot L$. The elements of matrix $\mathbf{A}=\left(a_{i j}\right)_{I \times J}$ can be computed knowing the positions of the squares in $W$ and the fans starting from the source points. The specialty of (3) is that the unknown vector $\mathbf{x}$ is binary, i.e., $x_{j} \in\{0,1\}$ for all $j=1,2, \ldots, J$.

In our model, the fan-beam integrals are measured by $L$ detectors placed opposite the sources uniformly along an arc having its center point in $S_{k}$ (Fig. 1). The arc of detectors is big enough

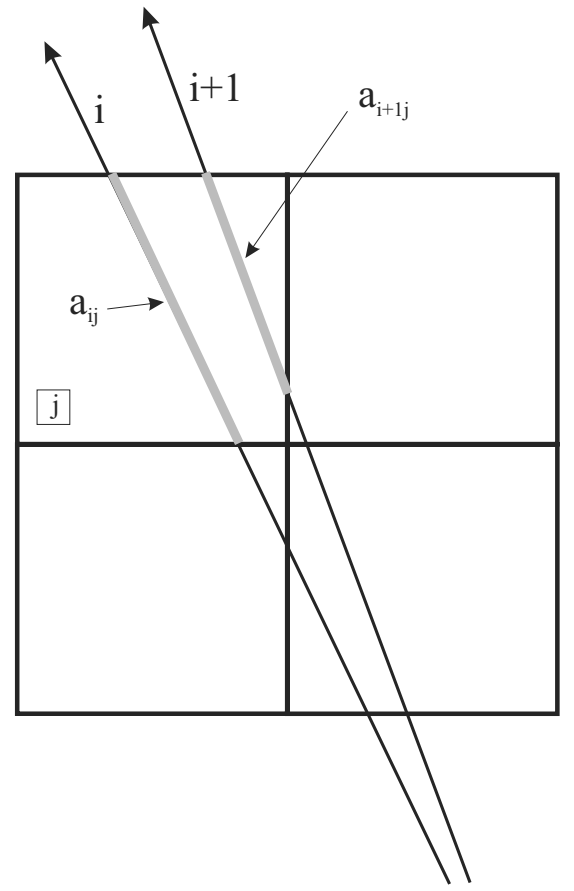

(a) Line integrals

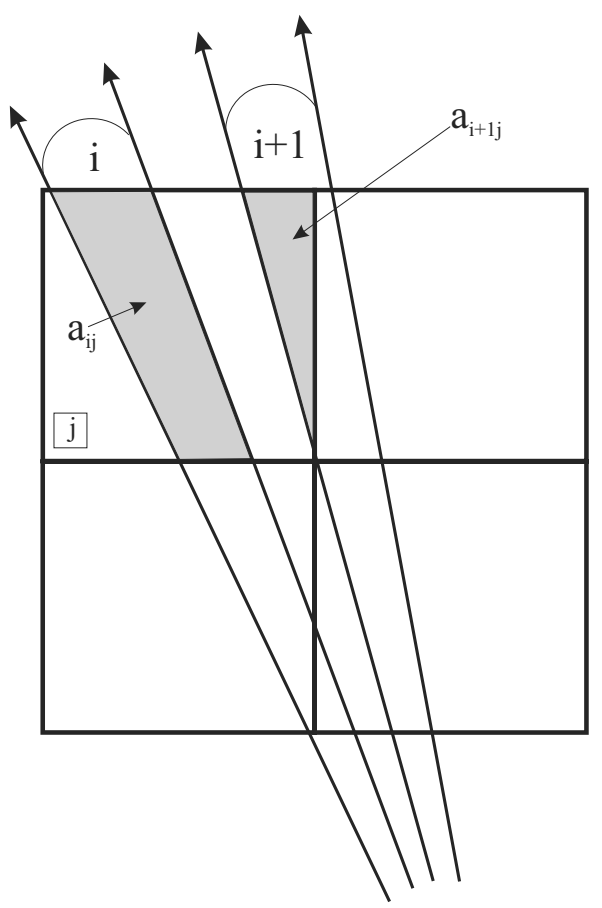

(b) Strip integrals

Fig. 2. Calculating the weights in the case of line and strip integrals. The gray $a_{i j}$ is the weight of the $j$ th unit square for the $i$ th fan. Equally, the gray $a_{i+1 j}$ is the weigth of the $j$ th unit square for the $(i+1)$ th fan. 
such that the whole image is between the halflines drawn from the source to the endpoints of the detector arc. Each detector measures one projection value. For simplicity, we assume that the center of the rectangle $W$ is in the origin $O$ of the coordinate system (Fig. 1).

The projection values are calculated in different ways in case of Eq. (3) for line and strip integrals:

- in the case of line integrals, the weights are given by the length of the section of the $i$ th half-lines and the $j$ th unit square of $W$ (Fig. 2(a)),

- in the case of strip integrals, the weights are given by the area of the section of the $i$ th beam and the $j$ th unit square of $W$ (Fig. 2(b)).

In real situations the projections are usually measured with a certain error. For this reason, Gaussian noise can be generated and added to the exact (analytically computed) projections for creating noisy projection data.

In our fan-beam model the following parameters can be varied.

$r$ : radius of circle $C_{r}$, i. e. the distance of the source points from the origin $O$;

$\theta_{0}$ : start angle determining the position of the first source point;

$K$ : number of source points;

$L$ : number of detector elements or, equivalently, the number of measurements from one source point;

$\alpha$ : fan angle;

$\eta$ : percentage of the additive Gaussian noise in the projections.

\section{Reconstruction by Optimization}

In order to solve the reconstruction problem FB in our fan-beam model we have to find a solution of the linear equation system

$$
\mathbf{A x}=\mathbf{b}
$$

such that $\mathbf{x}$ is binary. Since the number of projections is usually much smaller than the number of unknowns and the projections are known with some measurement error, it is more promising to try to find a binary $\mathbf{x}$, that satisfies (4) at least about.

Equation (4) can be reformulated as an optimization problem. Formally, find the minimum of the objective function

$$
C(\mathbf{x})=\|\mathbf{A} \mathbf{x}-\mathbf{b}\|+\gamma \cdot \Phi(\mathbf{x}),
$$

such that $\mathbf{x}$ is binary and $\Phi(\mathbf{x})$ is the regularization term with $\gamma$ regularization parameter. The regularization parameter is to weight the two terms in $C$. In the experiments we used a special kind of function for $\Phi_{\text {proto }}(x)$, namely

$$
\Phi(\mathbf{x})=\Phi_{\text {proto }}(\mathbf{x})=\sum_{j=0}^{n m-1} \operatorname{poz}\left(f_{j}-f_{j}^{(0)}\right),
$$

where poz denotes the positive part of $y$. Formally,

$$
\operatorname{poz}(y)=\left\{\begin{array}{l}
y, \text { if } y>0, \\
0, \text { otherwise }
\end{array}\right.
$$

and $f_{j}^{(0)}$ is a so-called prototype function. The prototype function is Fig. 4(b) for the Fig. 4(a) software phantom. As optimization method, we selected the Simulated Annealing (SA) [10].

\subsection{Simulated Annealing}

The SA method was implemented in the following way. (Fig. 3.)

The algorithm starts from an arbitrary initial binary image $\mathbf{x}^{(0)}$, an initial (high) temperature $T^{(0)}$ and calculates the objective function value $C(\mathbf{x})$. Then a position $j$ is chosen in the image $\mathbf{x}$ randomly. Let $\mathbf{x}^{\prime}$ be the image that differs from $\mathbf{x}$ only by changing the value of $\mathbf{x}$ in position $j$ to the other binary value, i.e., $x_{j}^{\prime}=1-x_{j}$. This change is accepted, i.e. $\mathbf{x}$ is replaced by $\mathbf{x}^{\prime}$ if $C\left(\mathbf{x}^{\prime}\right)<C(\mathbf{x})$. Even if the objective function does not get smaller, the change is accepted with a probability depending on the difference $\Delta C=C\left(\mathbf{x}^{\prime}\right)-C(\mathbf{x})$. Formally, the change is accepted even in this case if

$$
\exp (-\Delta C / \kappa T)>z,
$$

where $\kappa, T$ and $z$ are, respectively, the Boltzmann constant $\left(11.3805 \cdot 10^{-23}\right)$, the current temperature and a randomly generated number 


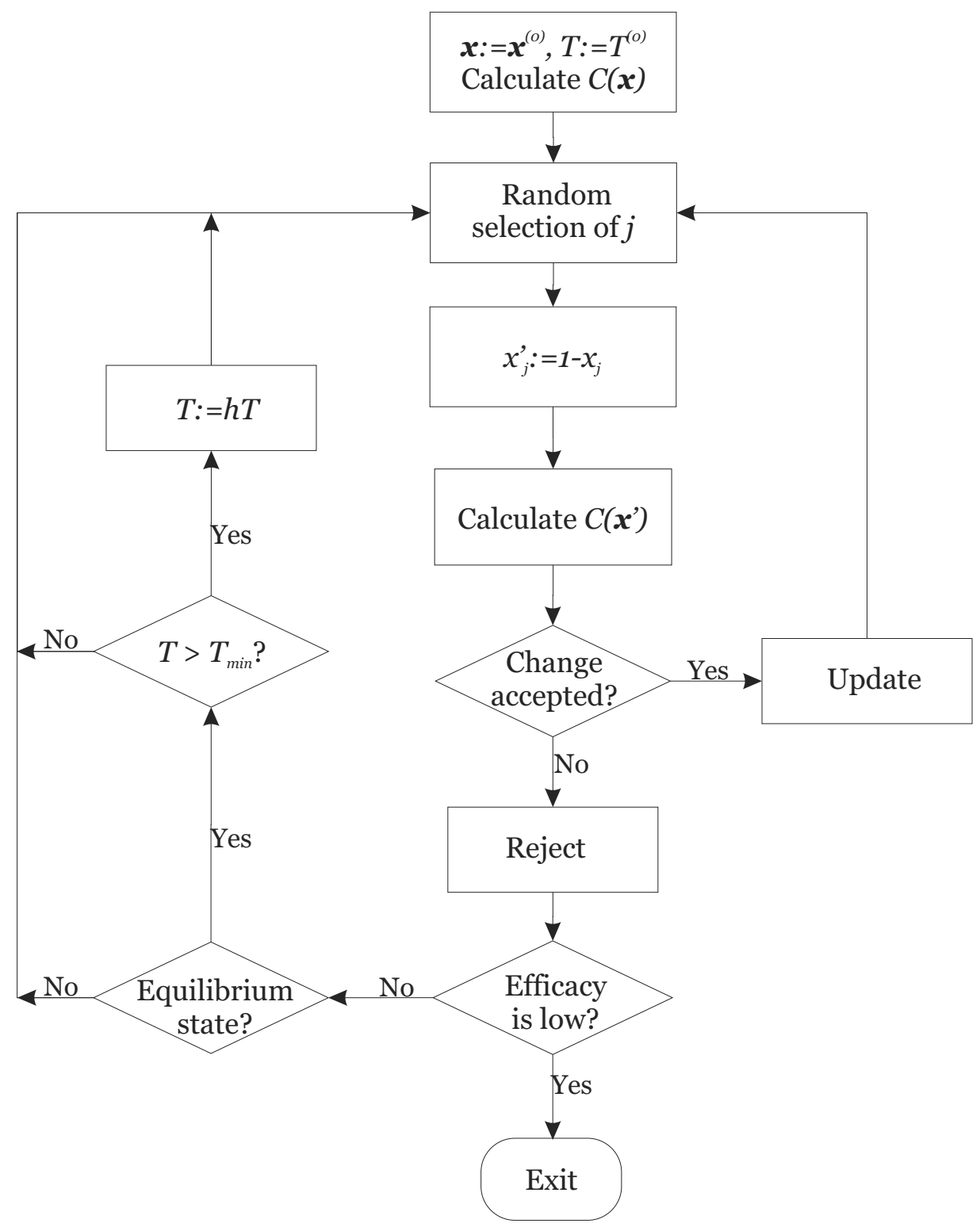

Fig. 3. Flow-chart of the implemented SA algorithm.

from the uniform distribution on the interval $[0,1]$. Otherwise, the change is rejected, i.e. $\mathbf{x}$ is not changed in this iteration step.

If a change is rejected, the level of efficacy of changes in the image in the last iterations is tested by counting the number of rejections in the last $N_{\text {iter }}$ iterations. If this number is greater than a given threshold value $R_{\mathrm{thr}}$, then the SA terminates. The temperature value is reduced if there are only very minor modifications in the value of the objective function $C(\mathbf{x})$ in the last iterations. This is measured as the variance of the cost function in the last $N_{\text {var }}$ iterations. Equilibrium state is reached if the estimate of the current $\Delta C$ variance is greater than the previous variance estimate.

If the equilibrium state is reached, the temperature $T$ is reduced (allowing changes when the value of the objective function is greater with smaller probabilities) and the algorithm continues with the lower temperature value $(T$ is replaced by $h \cdot T$, where $h$ is the so-called cooling factor).

In our SA algorithm we have set the parameters as follows: $\mathbf{x}^{(0)}=0$, i.e., empty image, $T^{(0)}=4.0, N_{\text {iter }}=10000, R_{\mathrm{thr}}=9990$, $N_{\mathrm{var}}=5000$, and $h=0.9$. 


\section{Results and Discussion}

The simulation experiments were performed with software phantom images having size of $200 \times 200$ (i.e., $n=200$ ). The projections of the phantom images (Fig. 4) were computed according to (3) for each parameter setting. The images were reconstructed from the projections by the SA algorithm described earlier. In order to get quantitative results, the original phantom images were compared with the reconstructed ones, pixel by pixel, according to the known relative mean error $[11,12]$

$$
M_{e}=\frac{\sum_{j=1}^{J}\left|x_{j}-\hat{x}_{j}\right|}{\sum_{j=1}^{J} \hat{x}_{j}},
$$

where $\hat{\mathbf{x}}=\left\{\hat{x}_{j}\right\}_{j=1}^{J}$ denotes the vector of the original image. Clearly, $M_{e} \geq 0$ and smaller value of $M_{e}$ indicates better agreement between $\mathbf{x}$ and $\hat{\mathbf{x}}$. Furthermore, $M_{e}=0$ if and only if $\mathbf{x}=\hat{\mathbf{x}}$.

Since we had an optimization method based on random-search, we repeated each test 100 times with the same parameter setting. The mean of the $100 M_{e}$ values have been computed and are presented later as the results of the tests with the given parameter setting.

Of course, several parameter settings were tested. One of them, the so-called baseline parameter setting, played a special role. The idea was that only one of the parameters was allowed to change at one time, the others remained the same as in the baseline parameter setting. In order to see the effect of the parameters on the quality of the reconstruction, we performed a sequence of tests for each parameter. For example, to see the effects of increasing the number of sources, we varied the value of $K$ in the model between 3 and 32, computed the projections for the same phantom image, ran the reconstruction algorithm with the same parameter settings 100 times, took the reconstructed 100 images, computed the $100 M_{e}$ values between the reconstructed and original images, and, finally, drew a curve showing the changes of $M_{e}$ as a function of the number of projections. The curve drawn from the mean values of such a sequence are going to be presented here as the final result

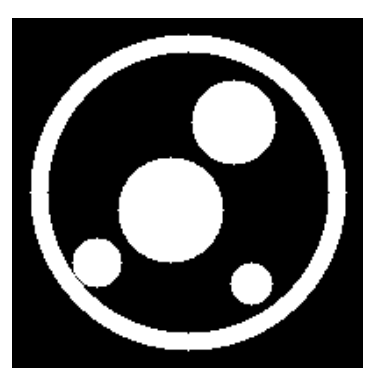

(a) Software phantom

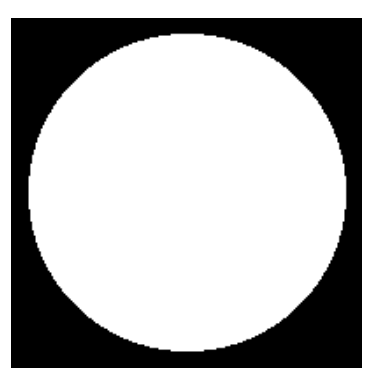

(b) Prototype function for the given software phantom
Fig. 4. Basic phantom image and prototype image used in the tests.

of the experiences connected with the selected parameter.

The baseline parameter setting, together with the range of the parameter values, is given in Table 1.

\begin{tabular}{|l|c|c|}
\hline Parameters & $\begin{array}{c}\text { Baseline } \\
\text { values }\end{array}$ & Range \\
\hline $\begin{array}{l}\text { Source and origin } \\
\text { distance }(r)\end{array}$ & 250 & {$[250,1750]$} \\
\hline $\begin{array}{l}\text { First source } \\
\text { position angle }\left(\theta_{0}\right)\end{array}$ & $0^{\circ}$ & {$\left[0^{\circ}, 360^{\circ} / K\right]$} \\
\hline $\begin{array}{l}\text { Number of } \\
\text { sources }(K)\end{array}$ & 32 & {$[2,32]$} \\
\hline $\begin{array}{l}\text { Number of detector } \\
\text { elements }(L)\end{array}$ & 401 & {$[101,401]$} \\
\hline $\begin{array}{l}\text { Gaussioan } \\
\text { noise }(\eta)\end{array}$ & $\eta \in\{0 \%, 5 \%\}$ & $\{0 \%, 5 \%\}$ \\
\hline $\begin{array}{l}\text { Fan angle for } \\
\text { strip integral }(\alpha)\end{array}$ & $(\varphi / L) \cdot 0.5$ & \\
\hline
\end{tabular}

Table 1. Baseline parameter setting.

In order to see the effect of noise, we repeated all tests not only with $0 \%$, but also with $5 \%$ noise. The results are presented as curves in the coordinate system of the studied parameter and the relative mean error. Accordingly, in the following subsections we are going to discuss the results as the effect of varying the parameters of the fan-beam model.

One of the most important problems we wanted to study were the effects of two different projection techniques. The first way is when projections are computed as integrals on fan-beam strips, i. e. on areas. The second one is when 
only the medial line is selected from the fanbeam strip and the projection is computed as the line integral along this line. For this reason, in the presentation of the results, there are two curves shown in each figure. One curve represents the changes in the case of strip integrals, while the other shows the results in the case of line integrals.

During the experiments we used $\gamma=145.0$ in the objective function (5), based on our previous experiences [9].

\subsection{Distance of the Source Point from the Origin}

This parameter, denoted by $r$, was changed between 250 and 1750 (the detector angle $\varphi$ and the fan angle $\alpha$ were also changed accordingly) (Fig. 5).

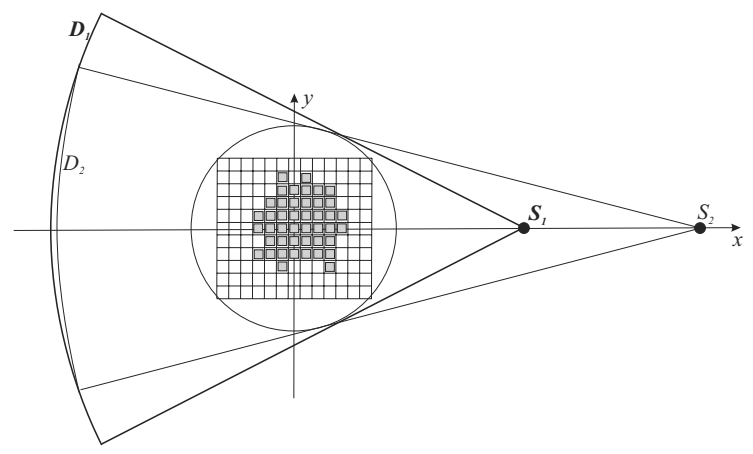

Fig. 5. Changing the distance between source and origin. Detector arc $\mathbf{D}_{\mathbf{1}}$ is for the source point $\mathbf{S}_{\mathbf{1}}$ and detector $\operatorname{arc} D_{2}$ is for the source point $S_{2}$.

That is, the fan-beam model is approaching the parallel projections by increasing $r$. The effect of this change is shown in Fig. 6.

Both curves in Fig. 6 show that there is no big difference between the results when the source is close to or far from the origin. More generally, there is no real difference between the fan-beam and parallel-beam projections if we change this distance. The Fig. 6(b) also shows that we got better results when we used line integrals to calculate the projections in the noisy case. In noisy experiments, the errors are higher in both cases.

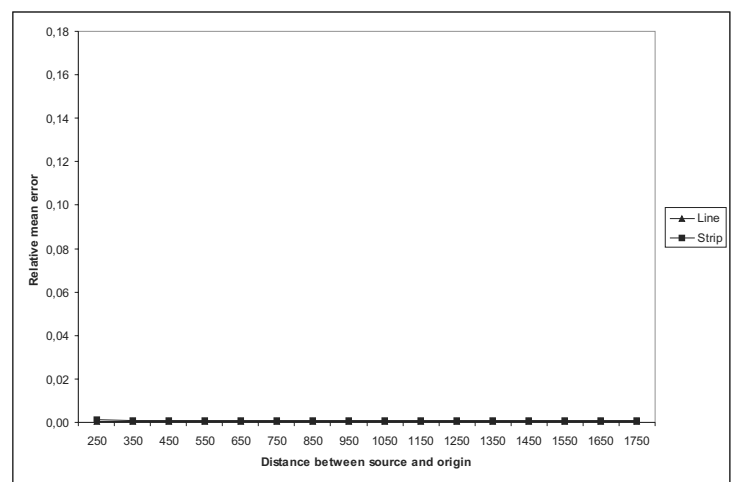

(a) Noiseless

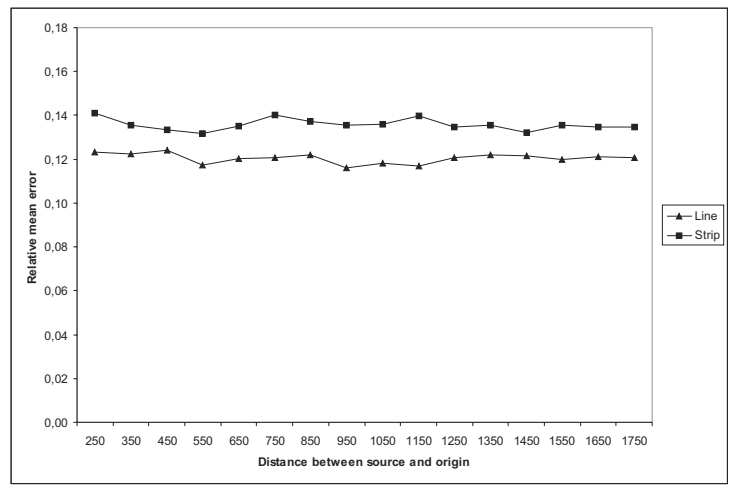

(b) $5 \%$ Gaussian noise

Fig. 6. Relative mean error as a function of the distance between source and origin $(r)$ for the line and strip integrals when $K=32$. There is no big difference between the results when the source is close to or far from the origin.

\subsection{Start Angle}

We changed the value of the angle parameter $\theta_{0}$ between 0 and $360 / K$ degrees. It is clear that by determining the position of the first source we determine also the positions of all sources along the circle $C_{r}$ (if the number of sources is fixed). Our previous results [9] show that there is no significant difference in relative mean square if we have relatively large number of source points/projections.

However, we got different curves if the number of projections was small and there was some special direction in the image. For example, when the number of source points was $K=4$, we got the curves presented in Fig. 7, showing that the quality of the reconstruction changes depending on the starting source position. 


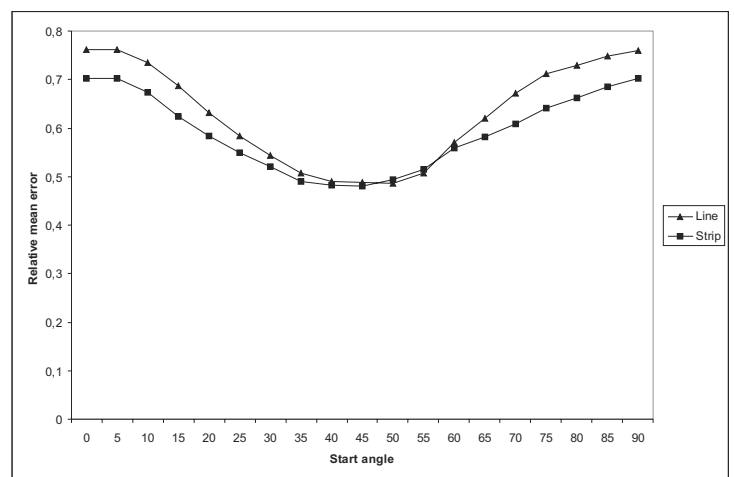

(a) Noiseless

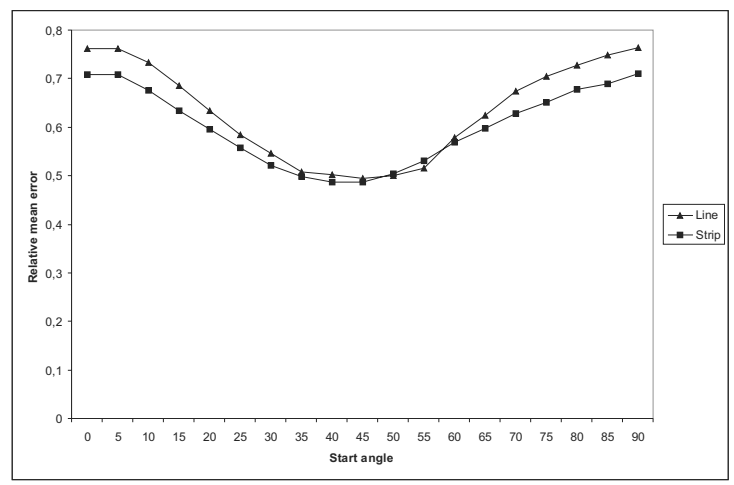

(b) $5 \%$ Gaussian noise

Fig. 7. Relative mean error as a function of the start angle $\left(\theta_{0}\right)$ for the line and strip integrals when $K=4$. We got the best result when the start angle was around $\theta_{0}=40^{\circ}$.

It is noticeable that start angle around $\theta_{0}=40^{\circ}$ gave the best result in both cases of line and strip integrals. The reason is that in this case the image (Fig. 4(a)) contains circles placed almost diagonally. In the curves corresponding to noisy projections this valley is also visible. We found that the relative mean errors were at the same level in noiseless and noisy cases as well.

Considering the average time duration, we can say that in certain points the reconstruction algorithm using strip integrals takes approximately twice the time compared to the one using line integrals (Fig. 8).

\subsection{Number of Source Points}

The number of source points $(K)$, in other words, the number of projections, was varied between 2 and 32 (Fig. 9). A natural expectation is that the quality of the reconstruction

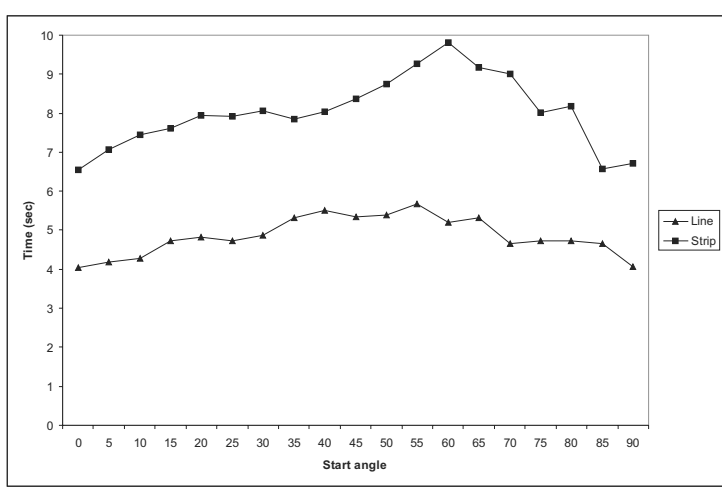

(a) Noiseless

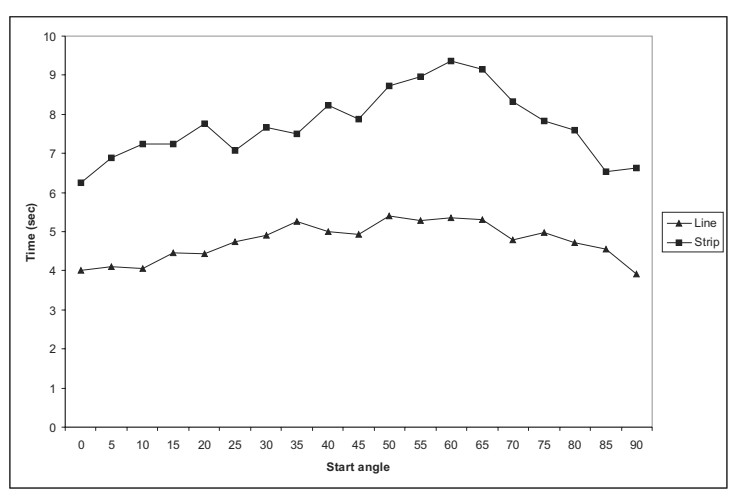

(b) $5 \%$ Gaussian noise

Fig. 8. Time ( $\mathrm{sec})$ as a function of the start angle $\left(\theta_{0}\right)$ for the line and strip integrals when $K=4$. Using strip integrals takes approximately twice the time in certain points compared to using line integrals.

improves by increasing the number of projections. The question here is, what is the (relatively small) number from which the quality changes very modestly.

The graphs in Fig. 9 show that the improvements is hardly recognizable beyond a certain value of $K$.

In noiseless case, if we had sufficient number of equations for the unique determination of the linear equation (3), we reached the solution at $K=20$ when we used strip integrals and $K=22$ when we used line integrals. In the noisy case line integrals gave better result from a certain number of sources.

In this experiment we can notice that the reconstruction always takes longer using strip integrals than using line integrals (Fig. 10). The reason can be the smoothing effect of the strip integrals. 


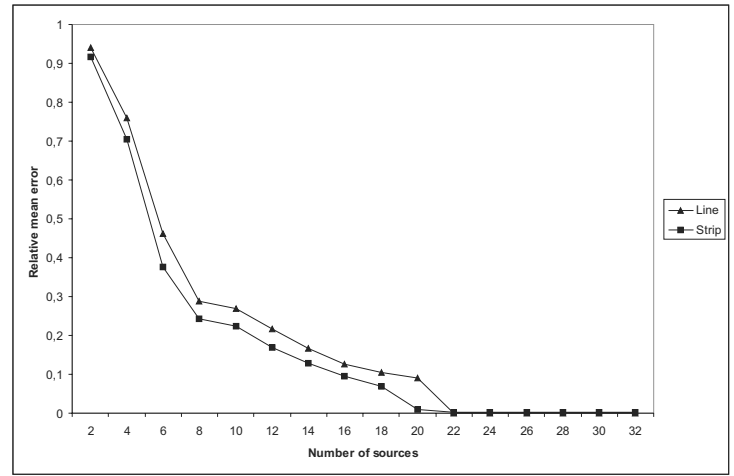

(a) No noise

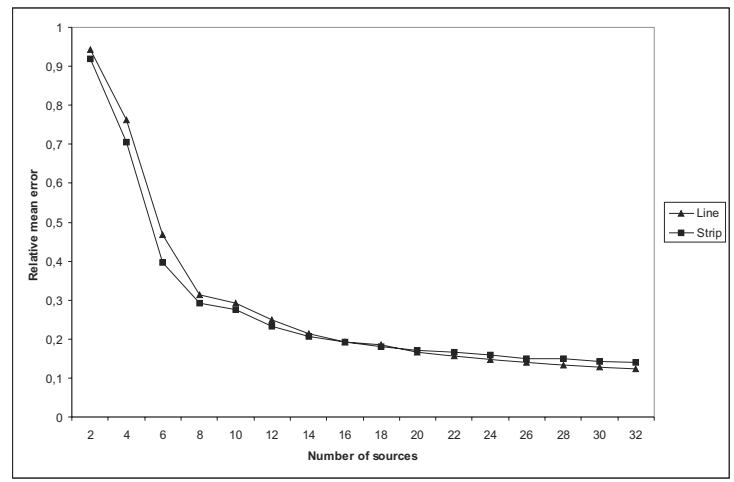

(b) $5 \%$ Gaussisan noise

Fig. 9. Relative mean error as a function of the number of sources $(K)$ for the line and strip integrals. We reached the solution at $K=20$ when we used strip integrals and $K=22$ when we used line integrals. In the noisy case, line integrals gave better result from a certain number of sources.

\subsection{Number of Detector Elements}

It is clear that if we have more detector elements $(L)$, we also have more equations in $(3)$ and, therefore, more information about the image. However, we cannot get better mean square error (Fig. ??(a)) beyond a certain value of L, here $L=261$.

In the noisy case, the quality of the reconstruction is improving almost everywhere in the parameter interval (Fig. ??(b)). As a summary, we can say that simply by increasing the number of detector elements we reached substantial improvements only up to a certain $\operatorname{limit}(L \approx n)$.

\section{Discussion and Conclusions}

We have studied the geometric parameters of the fan-beam projections used in the recon-

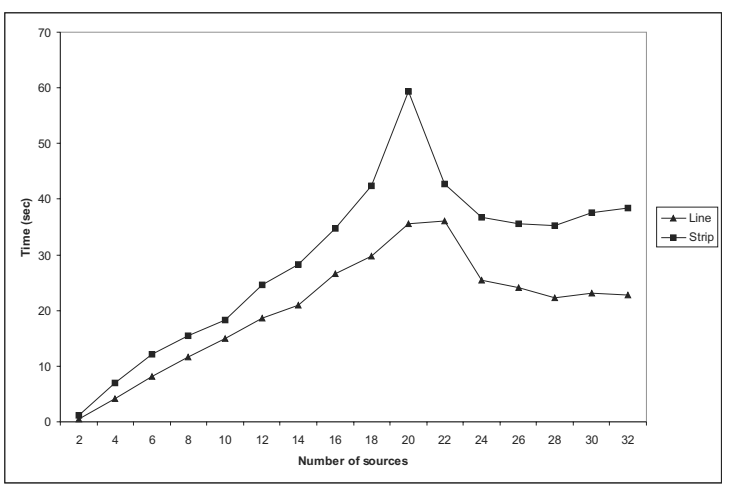

(a) No noise

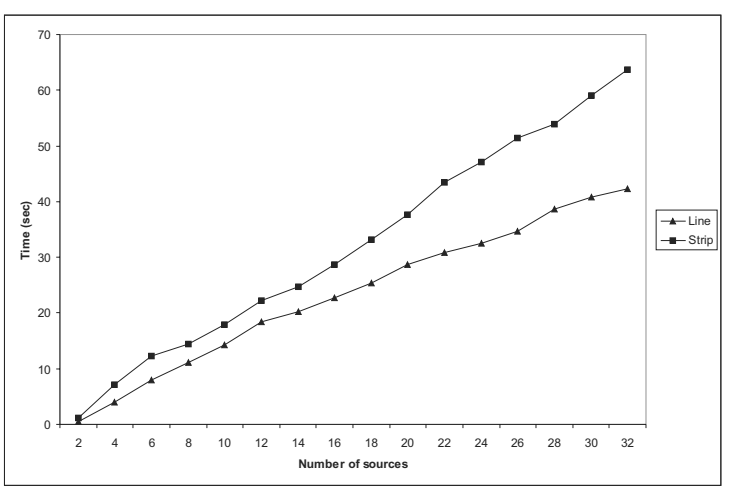

(b) $5 \%$ Gaussisan noise

Fig. 10. Time (sec) as a function of the number of sources $(K)$ for the line and strip integrals. The reconstruction takes longer using strip integrals.

struction of binary matrices. Simulation experiments were performed by reconstructing phantoms with different parameter settings.

We observed no remarkable difference between the line and strip integrals in the noiseless case when the distance between the source positions and the origin was large enough. The reason for the small differences between using strip and line integrals probably comes from the smoothing effect of the strip integral. If we have small number of sources, the start angle plays significant role in the quality of the reconstruction in case of the given phantom.

In all other experiments, the differences caused by the two kinds of integrals in the computation of the projections were very small too. For the noiseless (ideal) case and for the noisy case, it is sufficient to compute the projections as line integrals instead of the computationally more expensive strip integrals that would take approximately twice longer. 


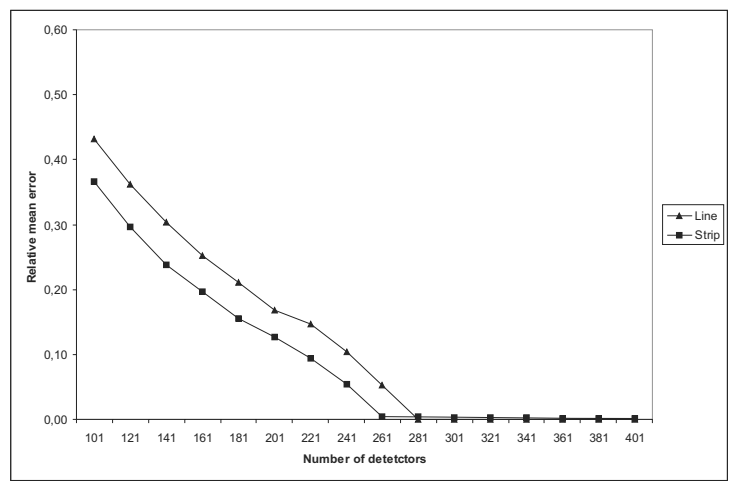

(a) No noise

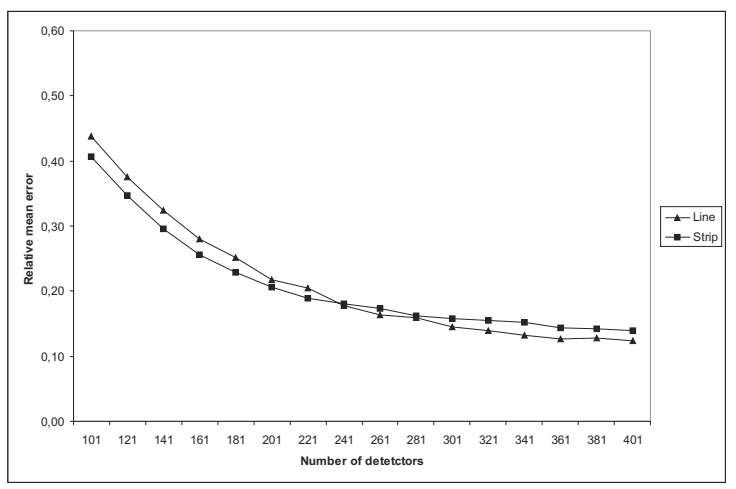

(b) $5 \%$ Gaussian noise

Fig. 11. Relative mean error as a function of the number of detector elements $(L)$ for the line and strip integrals when $K=32$. By increasing the number of detector elements we reached substantial improvements only up to a certain limit $(L \approx n)$.

The final aim of these experiments was to get good parameter settings in a discrete tomography system to be realized in future applications. The results presented here can be used in planning such physical imaging devices.

\section{Acknowledgment}

This work was supported by the OTKA grant T 048476 and NSF grant DMS0306215 (Aspects of Discrete Tomography).

\section{References}

[1] G.T. HeRman, A. KuBA, Discrete Tomography: Foundations, Algorithms, and Applications. Birkhauser, Boston, (1999).

[2] R.A. BRUALDI, Matrices of zeros and ones with fixed row and column sum vectors, Linear Algebra AppI. 33 (1980) pp. 159-231.
[3] A. Kuba, G.T. HeRman, A historical introduction. In [1], (1999).

[4] A.C. KAK, M. SLANEY, Principles of Computerized Tomographic Imaging. New York, NY: IEEE Press, Inc., (1988).

[5] P. GRAngeat, Mathematical framework of cone beam 3D reconstruction via the first derivative of the Radon transform. Mathematical Methods in Tomography, Lecture notes in Mathematics, eds. G.T. Herman, A.K. Louis, and F. Natterer, 1497 (1991), pp. 66-97.

[6] B. Chalmond, F. Coldefy, B. LaVayssiere, Tomographic reconstruction from non-calibrated noisy projections in non-destructive evaluation. Inverse Problems 15 (1999), pp. 399-411.

[7] A. KubA, L. Ruskó, L. RodeK, Z. Kiss, Preliminary results of Discrete Tomography in Neutron Imaging. IEEE Trans. Nucl. Sci. 52 (2005), pp. 380-385.

[8] N. Robert, F. PEYRIN, M.J. YAFFe, Binary vascular reconstruction from limited number of cone beam projections. Med. Phys. 21 (1994), pp. 1839-1851.

[9] A. NAGY, A. KUBA, Reconstruction of binary matrices from fan-beam projections. Acta Cybernetica 17 (2005), pp. 359-383.

[10] N. Metropolis, A. Rosenbluth, M. RosenBLUTH, A. TELlER, AND E. TEller, Equation of state calculation by fast computing machines. J. Chem. Phys. 21 (1953), pp. 1087-1092.

[11] A. Kuba, G.T. Herman, S. Matej, A. TodDPOKROPEK, Medical applications of discrete tomography, in DIMACS Series in Discr. Math. and Theor. Comp. Sci. 55 (2000), pp. 195-208.

[12] G.T. Herman, A. KuBA, Discrete tomography in medical imaging, Proc. of IEEE 91 (2003), pp. 1612-1626.

Received: December, 2004 Revised: February, 2006; March, 2006 Accepted: April, 2006

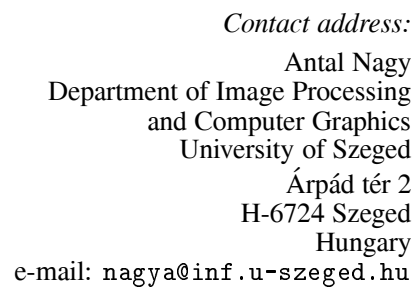

ANTAL NAGY is a research assistant at the Department of Image Processing and Computer Graphics, University of Szeged. His areas of interest include image processing, discrete tomography, and Picture Archiving and Communication Systems.

Attila Kuba is a Professor at the Department of Image Processing and Computer Graphics, University of Szeged, Hungary. His areas of interest include medical image processing and discrete tomography. $\mathrm{He}$ is a member of IEEE Signal Processing Society. 